\title{
Informe final de las prácticas de profesionalización como docente de Lengua y Literatura Hispánicas
}

\section{Final report of the professionalization practices as a teacher of Hispanic Lan- guage and Literature}

(C) UNAN-Managua

Recibido: 24 de junio 2021

Aprobado: 24 de julio 2021
Daniel Francisco Zamora Sotelo

https://orcid.org/0000-0002-8211-7688

larreguichad@gmail.com

\section{RESUMEN}

Las prácticas de profesionalización son para el estudiante de Lengua y Literatura Hispánicas el último paso de apenas la primera etapa de su permanente aprendizaje como docente egresado de la UNANManagua; una actuación que consta de la búsqueda diaria de información y el empleo de nuevas estrategias en el aula de clases para lograr un proceso de aprendizaje-enseñanza de mayor preponderancia en la educación media de Nicaragua.

Palabras clave: profesionalización, lengua y literatura, Universidad, prácticas.

\section{SUMMARY}

The professionalization practices are for the student of Hispanic Language and Literature the last step of just the first stage of his permanent learning as a teacher graduated from UNAN-Managua; an action that consists of the daily search for information and the use of new strategies in the classroom to achieve a learning-teaching process of greater preponderance in secondary education in Nicaragua

Keywords: professionalization, language and literature, University, internships.

Como un último acercamiento a este contexto por parte de la casa de estudios superiores, se resalta el control de un grupo de secundaria, con tareas docentes que implican el uso de las distintas metodologías de enseñanza y evaluación, además de la aplicación del conocimiento científico para la proyección del contenido sugerido por la Macro Unidad Pedagógica (MUP) del MINED, que ha venido en sustitución de la Malla Curricular a raíz de la situación sanitaria global creada por la pandemia del COVID-19.

Son muchos los inconvenientes que existen en el campo de la educación. Muchos aparecen a simple vista; otros, se conocen en el trayecto de la labor docente, sin embargo, el sistema está comprendido por todos sus agentes: autoridades estatales, familia-comunidad y, por supuesto, estudiantes y docentes. Encontrar entre estos a un solo culpable de las carencias del aprendizaje no es objetivo, de modo que se insta a una autocrítica de cada uno de los actores del statu quo para su mejoramiento.

Dicho lo anterior, se ha creado este informe para el último seminario de la clase "Prácticas de profesionalización", el cual es una reflexión a partir de la elaboración del instrumento FODA, matriz que muestra las forta- 
lezas, oportunidades, debilidades y amenazas del docente practicante. Como aclaración, este informe, si bien está escrito de forma impersonal, se basa en la experiencia propia.

\begin{tabular}{|c|c|}
\hline Fortalezas & Oportunidades \\
\hline 1. Competencias científicas & $\begin{array}{l}\text { 1.1. Desarrollo de nuevo conocimiento pertinente para el estudiante. } \\
\text { 1.2. Búsqueda de más conocimiento como docente. }\end{array}$ \\
\hline 2. Competencias metodológicas & $\begin{array}{l}\text { 2.1. Seguimiento del conocimiento adquirido de los estudiantes. } \\
\text { 2.2. Competencia investigativa más significativa en el campo de la educación. }\end{array}$ \\
\hline 3. Competencias humanistas & 3. Capacidad de adaptabilidad con cualquier grupo de estudiantes. \\
\hline Debilidades & Amenazas \\
\hline $\begin{array}{l}\text { 1. Currículum vitae no correspon- } \\
\text { diente a la edad }\end{array}$ & $\begin{array}{l}\text { 1. Poca o nula probabilidad de ejercer la licenciatura de Lengua y literatura en el } \\
\text { sistema público o privado }\end{array}$ \\
\hline
\end{tabular}

Tabla 1

Matriz FODA

\section{Descripción y análisis del instrumento FODA de la experiencia docente en las prácticas de profesionalización}

El FODA presenta en primer lugar las fortalezas, que en las prácticas de profesionalización se destacaron y afianzaron: las competencias científicas, metodológicas y humanistas. El Departamento de Español de la UNAN-Managua prepara en estas áreas a sus estudiantes para que la docencia que ellos ejerzan sea óptima y responsable, un reflejo de la educación recibida en la alma mater.

Las clases que implican la ciencia de la literatura resultan en un ejercicio intelectual de pleno disfrute. La adquisición de este conocimiento fue relativamente fácil. La pasión por aprender literatura se refleja en la pasión por enseñar literatura, y no menos enérgica es la actitud al enseñar otros contenidos que le competen a la carrera.

Por otro lado, para las competencias metodológicas, aunque su obtención fue más laboriosa, no son pocas las clases que integradas lograron su desarrollo: Investigación documental, Metodología de la investigación, Seminario de graduación PEM, Didáctica de la lengua y la literatura e Investigación aplicada. En el escenario de las prácticas de profesionalización, el planeamiento de una secuencia didáctica, sus técnicas e instrumentos para la recopilación de datos y otros elementos metodológicos exponen las problemáticas y la necesidad del autoajuste del método mismo para la enseñanza.

Luego, se muestran las competencias humanistas, que son consideradas también parte de una metodología, se basan más en la actitud individual del estudiante de la carrera de Lengua y Literatura que una clase más del pensum curricular. En este caso se expresa la capacidad para relacionarse con el grupo de secundaria por 
medio de un alto grado de empatía. De ninguna manera esto debe ser visto como una debilidad como han sugerido docentes adaptados al mal sistema; tampoco se refiere solamente a la parte afectiva, sino también a la facultad para lograr la adaptación de participaciones tímidas o vagas de los estudiantes a participaciones convenientes para la clase.

De todas estas fortalezas se crean las oportunidades para el progreso de la educación, tanto para el profesor como para el estudiante. Por un lado se tiene el desarrollo de nuevo conocimiento pertinente para el estudiante; y por otro, la oportunidad de búsqueda de más conocimiento como docente, la cual es una tarea obligatoria para los egresados de la carrera, pues las nuevas tecnologías y la globalización requieren de un pensamiento en actualización, porque hasta las ideas toman formas distintas con el paso del tiempo.

Posteriormente, desde el punto de vista metodológico, existe la oportunidad de continuar la evaluación del conocimiento adquirido de los estudiantes, para saber cuán ideal fue o cuánto necesita mejorar durante el resto de su preparación; lo que al docente le ofrece, a la vez, la oportunidad de tener una competencia investigativa más significativa en el campo de la educación, ya que los datos que revelan las problemáticas son mejor observables con cada experiencia, con cada año integrado en el sistema educativo.

Seguidamente, se tiene la capacidad de adaptabilidad con cualquier grupo de estudiantes. Esta oportunidad ligada a la competencia humanista, acompaña a la pasión de enseñar literatura mencionada párrafos atrás. No sirve de mucho la enseñanza de la literatura si se hace únicamente desde su enfoque historicista; debe complementarse con el enfoque comunicativo y estético, y un docente que no conecta con sus estudiantes, difícilmente obtiene de ellos comunicación y el reflejo del gusto que él tiene por la literatura. Los estudiantes deben sentir un ambiente de confianza donde sepan que sus ideas tienen valor.

En otro orden, el FODA también abarca las debilidades del docente en progreso. De estas se ha de señalar principalmente la de un currículum vitae que no es correspondiente a la edad. Del mismo modo, tanto a profesores jóvenes como mayores (caso personal), el sistema de educación exige por encima de la aptitud, una experiencia formal frente a la clase, que equivocadamente cree que no se obtiene de las prácticas de familiarización, especialización y profesionalización. Se trata de un obstáculo que no debería existir para ejercer la docencia.

Lo pospuesto resulta en una dicotomía entre la pasión y el sentimiento de autoinfravaloración, que deviene en la amenaza de creer que se está ante la poca o nula probabilidad de ejercer la licenciatura de Lengua y literatura en el sistema público o privado del país, sin importar talento, competencias y conexiones laborales. A lo largo de todas las prácticas de profesionalización este concepto resulta en una carga psicológica aunada a las que un experto en salud mental puede tratar ocasionalmente.

En conclusión, las prácticas de profesionalización contribuyen en gran medida a la formación del futuro docente. Claro está, sigue siendo un acercamiento a un oficio que demanda la preparación constante debido a la gran can- 
tidad de problemáticas que existen, pero que en esta experiencia se aprecian mejor que en otras prácticas de la carrera gracias a la inmersión total que el Departamento de Español de la UNAN-Managua solicita en este penúltimo semestre de quinto año.

Bien es cierto que las carencias del sistema educativo son evidentes, como lo son las propias dificultades que el docente practicante tiene y que se convierten en amenazas. No obstante, un análisis de FODA también muestra las fortalezas y las oportunidades para que, en términos de Weihrich, se puedan utilizar las estrategias de maxi-maxi, para maximizar fortalezas y así maximizar oportunidades; maxi-mini, para maximizar las fortalezas y de este modo minimizar las amenazas; mini-maxi, para minimizar las debilidades y con esto maximizar las oportunidades; y mini-mini, para minimizar las debilidades y finalmente minimizar las amenazas expuestas en este informe. 